\title{
Psychological transformation methods and Countermeasures of higher vocational students with mathematics learning disabilities
}

\author{
Daquan Chang* \\ WuXi City College of Vocational Technology, Wuxi 214000, Jiangsu, China.
}

\begin{abstract}
In recent years, the learning disabilities of vocational school students for their weak mathematical foundation and lack of interest have become a hot topic in vocational education. Mathematics learning disabilities of vocational school students are not only objective learning difficulties, but also subjective learning psychology problems. Exploring the psychology transformation methods and countermeasures of mathematics learning disabilities of higher vocational students provides an important reference for solving this problem.
\end{abstract}

Keywords:learning disabilities;psychology; transformation methods

Fund project:2020 WuXi City College of Vocational Technology Scientific Research Project: Research on the Psychological Assistance Mechanism of Higher Vocational Students' Mathematics Tiredness Dilemma(NO.WXCY-2020-KY-20)

In recent years, the number of students in my country's higher vocational colleges has continued to expand, the entrance scores of students are not high, and the students' basic cultural knowledge is generally poor. Most students feel embarrassed by the advanced mathematics, economic mathematics and other courses offered by the school. They are generally struggling to study and have low interest. After a period of study for newly enrolled vocational students, if their mathematics learning difficulties cannot be resolved in time, they will often fall into serious mathematics learning obstacles. This situation will greatly affect students' learning effects on subsequent professional courses based on mathematics, and also hinder the improvement of the quality of talent

Copyright (C) 2020 Daquan Chang

doi: 10.18282/1-e.v9i4.1707

This is an open-access article distributed under the terms of the Creative Commons Attribution Non-Commercial License (http://creativecommons.org/licenses/by-nc/4.0/), which permits unrestricted non-commercial use, distribution, and reproduction in any medium, provided the original work is properly cited.

is possibility to prevent it, but whether it happens or not depends on Russia. If Russia makes a concession that Kremlin will not allow Snowden to take refuge in Russia, the White house will not cancel the summit then there will not be any problems between these two countries. The analysis of present progressive in report can help us understand that the White House hopes that Russian can refuse to help Edward Snowden. On one hand, this news should report facts, but at the same time, the standpoint requires the description to have the function of alerting. On the other hand, present progressive softens the voice of the decision made by the USA, and indirectly tell readers that what they will do is not their true will. If the decision has been made, Russia should take responsibility for it.

\section{Conclusion}

From what has been analyzed above, we can know that the usage of modal verbs and tense can help us dig up the attitudes of the reporter or the side he stands for. Different modal verbs will describe different feelings. Correct usage of them can avoid absoluteness and let readers accept the information easily. Besides, the functions of tense cannot be limited in time, they also can realize the interpersonal function in some certain context.

\section{Bibliography:}

[1]Thompson G.Introducing Functional Grammar[M]. Beijing: Foreign Language Teaching and Research Press ,2000:28

[2]He Wei. modal usage of tenses: grammatical metaphors [J]. foreign language and foreign language teaching ,2008,(7).

[3]He Wei. A Comprehensive Study of Foreign English Temporal Studies [J].]1 Journal of PLA Foreign Language Institute, $\mathrm{a}(1) .2006$

[4]He Wei. A Study on Systemic Functional Grammar of Tense Clauses [J].] Hierarchy Foreign Language and Foreign Language Teaching, b(8).2006

[5]Ginna. A Study on the Evaluation Function of Tenses Foreign Language and Foreign Language Teaching ,2009(8):24-25...

[6]Xie Wei. A Study on the Meaning of English Modal Verbs Journal of Hunan Institute of Humanities and Science and Technology ,2007,(2)

[7]Zhao Pu. A Semantic and Pragmatic Analysis of the Meaning of Modal Auxiliary Verbs in English [J].]1 Foreign Language Studies,2004,(2). 
training in higher vocational colleges. We recognize that mathematics learning disabilities of higher vocational students are not only objective and practical learning difficulties, but also subjective learning psychological barriers. Exploring and studying the psychology transformation methods and countermeasures of mathematics learning obstacles of higher vocational students is an important way to solve this problem.

\section{The connotation and cause of mathematics learning obstacles of vocational students}

In order to understand the psychological barriers of higher vocational students in mathematics learning, we selected students from the Department of Mechanical and Electrical Engineering and the Department of Construction Engineering of Wuxi City Vocational and Technical College etc.to conduct investigations and studies. According to the freshmen's college entrance examination scores, the mathematics test scores after enrollment, and the basic situation of students' learning learned by the head teacher and class teachers, the important characteristics of vocational students' psychological obstacles in mathematics learning are derived, which are mainly manifested in the following six aspects. First, it is difficult for higher vocational students to adapt to the college mathematics teaching style. The main difference between students with mathematics difficulties and those students with excellent math scores is their adaptability to mathematics teaching methods. The weak mathematical foundation of students is obviously the main reason for this difference. Second, there is no motivation in mathematics learning, and indifferent learning interest is high. Common characteristics of students with poor vocational mathematics; third, lack of motivation in mathematics learning, unable to combine mathematics learning content with learning objectives; fourth, vocational students show insufficient confidence when they encounter difficulties in learning mathematics, and withdraw from difficulties. Fifth, students with poor mathematics scores in higher vocational colleges lack a sense of achievement in mathematics, and learning difficulties generally leave students with a bad psychological experience; sixth, many vocational students are inefficient in learning mathematics and do not pay attention to the methods and strategies of mathematics learning .

The causes of vocational students' mathematics learning obstacles are mainly reflected in the following aspects: 1 . The students have a weak cognitive foundation of the mathematics knowledge system and poor cognitive structure; 2 . The students do not agree with the role and influence of mathematics learning goals on future career planning ; 3 . Teachers' professional quality is insufficient; 4. The existing evaluation system of higher vocational mathematics courses is outdated and cannot adapt to changing new academic conditions.

\section{A review of researches on psychology transformation methods of mathematics learning disabilities}

The root causes of mathematics learning disabilities are students' weak mathematics foundation, poor learning methods and low learning efficiency. These can be summarized as difficulties in mathematics learning ${ }^{[1]}$. The concept of learning disability (LD for short) was first proposed by the famous British educator Kirk in 1963. Since the 1960s, learning disabilities have always been one of the hot issues studied by foreign education researchers. Chinese scholars began to study the problem of learning difficulties only in the 1990s gradually. The definition of mathematics learning disability requires a clear definition and explanation of the core defects of mathematics learning disability, which is a common consensus of domestic and foreign researchers. According to the psychological investigation of learners and the completion of the set arithmetic tasks, Geary proposed a subtype model of MD. Domestic scholars have continuously enriched the research results of mathematics learning difficulties, but the depth and breadth of research needs to be further improved. In 2001, Du Yuxiang gave the definition of learning difficulties. He believed that "poor academic performance and persistent learning disabilities" were the essential characteristics of learning disabilities. Xu Xingchun believed that poor cognitive structure, lack of problem-solving strategies, lack of divergent thinking, and low self-evaluation effectiveness are the main manifestations of mathematics learning difficulties. In 2009, Wang Changgui conducted a research on the problem of mathematics learning disabilities of vocational students. He believed that mathematics learning difficulties of vocational mathematics students refer to students' difficulties in adapting to mathematics learning, which are manifested in lack of motivation, lack of motivation, poor learning methods and strategies, and academic performance low. Du Qingkun and Xu Chuansheng have done a lot of work on the transformation of mathematics learning disabilities. They gave five countermeasures to transform students with mathematics difficulties. In 2013, Dong Lihua applied mathematical statistics methods and combined with educational psychology to study the transformation of mathematics learning difficulties and made a lot of achievements. She proposed measures such as rectification of teaching content, improvement of teaching methods, and reconstruction of assessment methods to promote the transformation of students with mathematical difficulties. The above studies have achieved certain results, but the pertinence and operability of the research need to be further strengthened. Therefore, how to build a scientific, systematic and easy-to-operate psychology transformation method and strategy based on the characteristics of mathematics subjects and the psychological performance of vocational students in combination with teaching practice, and successfully transform students with learning difficulties in vocational mathematics, is important for our attention and research Subject.

\section{Various Countermeasures of Improve the Psychology Transformation Methods of Mathematics Learning Obstacles}

By analyzing the connotation and causes of mathematics learning disabilities of higher vocational students, we can formulate and improve the methods and countermeasures of psychological transformation of mathematics learning disabilities.

First of all, improving students' psychological experience in mathematics learning is a prerequisite for establishing a psychological assistance mechanism for students with mathematical difficulties ${ }^{[2]}$. By adjusting learning content, improving evaluation methods and other means to improve the learning motivation of mathematics-weary students, enhance students' interest in learning, and improve students' psychological experience of learning mathematics. Second, focusing on individual differences 
to teach students in accordance with their aptitude is an important principle for the psychological transformation of students who have mathematical difficulties. Students with mathematics learning difficulties have obvious individual differences and strong independence, and their focus and interest points are different. Therefore, according to each person's different situations and characteristics and strictly follow the principle of teaching students in accordance with their aptitude, they can achieve better psychological transformation effects. Third, enhancing students' sense of accomplishment and gain is the key to perfecting the psychological transformation mechanism of students with mathematical difficulties. It is a common phenomenon for students who are in difficulties in mathematics to be marginalized. If teachers do not properly teach students with mathematics difficulties, their confidence will often be hit hard. Therefore, we should create a good environment for students with mathematical difficulties to benefit from practice and enhance their sense of accomplishment in their learning effectiveness. Fourth, create a good interactive atmosphere for teaching and provide psychological and emotional support for math-weary students. Students with difficulties in mathematics learning are generally afraid of difficulties in communicating with teachers. Negative emotions such as low self-esteem, self-blame, and helplessness will undoubtedly further aggravate their learning difficulties. Therefore, truly caring about students, channeling negative emotions, and creating a good teacher-student emotional interaction atmosphere is of great significance to perfecting the psychology transformation mechanism of students with mathematical difficulties.

\section{Constructing a Scientific System of Psychological Transformation Methods for Mathematics Learning Disabilities of Higher Vocational Students}

First, combine learning motivation with career planning education to promote the formation of a benign incentive mechanism for mathematics learning motivation. Students lack the motivation to learn mathematics. One of the important reasons for the lack of motivation is that mathematics learning cannot be combined with reality and cannot be connected with future career plans. In the actual teaching work, teachers should further refine the teaching materials, adjust the teaching content, and strengthen the students' mathematical humanistic accomplishment and mathematical quality training. Through the teaching of mathematics culture and history of mathematics, students can truly realize the positive significance of mathematics to life and society from the bottom of their hearts, so as to stimulate students' learning motivation and promote the formation of a benign incentive mechanism for learning motivation. Second, attach importance to interactive teaching and overcome the psychological barriers of students who are tired of learning mathematics. Teachers should carefully optimize the classroom teaching design and increase the frequency of communication and interaction with math-weary students. By setting up a variety of small learning tasks with low difficulty and strong interaction, students with mathematics difficulties can continue to enhance their sense of learning, and gradually overcome the psychological obstacles of learning tiredness and fear of learning. Third, implement various measures for psychological transformation of students with mathematical difficulties to ensure that students with mathematical difficulties complete their learning goals. Teachers should help students formulate learning goals within their ability according to the specific situation of students. Attach importance to feedback and evaluation in the process of students achieving their learning goals, and strengthen their successful learning experience by giving them positive evaluations. The above-mentioned goals, evaluations, and experiences are the key elements of psychological transformation methods for students with number difficulties[3]. Fourth, strengthen the education of students' view on mathematics and improve their humanistic qualities in mathematics. The individual's fundamental view and understanding of mathematics is the view of mathematics. The mathematics outlook of vocational students is affected by many factors such as their own mathematics foundation, elementary and middle school mathematics education, social environment, family environment and so on. There is no doubt that a correct view of mathematics will greatly promote students' mathematics learning. The mathematics view of the students with mathematics difficulties in higher vocational colleges is often distorted and unhealthy. The viewpoints of inability to learn mathematics and useless mathematics are common among students with mathematics difficulties in higher vocational schools. Therefore, reshaping the healthy view of mathematics of vocational students and cultivating the humanistic literacy of mathematics of vocational students is an important task of the transformation mechanism of mathematics learning disabilities psychology ${ }^{[4]}$.

\section{References:}

[1] Bao Jiping, Han Yuqiang. Attribution and countermeasures of psychological barriers in learning[J]. Educational Exploration, 2001, (11).

[2] Wang Chong, Sun Fengzhi, Sha Yuanxia. Psychological obstacles and countermeasures for students in higher mathematics classrooms in applied technology colleges [J].

Journal of Chifeng University: Natural Science Edition, 2015, (6).

[3] Zhang Guimei. Higher mathematics teaching should pay attention to the cultivation of students' non-intelligence factors [J]. Educational Exploration, 2012, (4).

[4] Yang Shu, Sun Shiguo, Wen Xiaoyan. Analysis of the psychological barriers of engineering students in learning advanced mathematics [J]. Education and Occupation, 2008, (13).

[5] Pan Changxiang, Li Changhu. Some thoughts on psychological barriers to learning[J]. Basic Education Research, 1995, (1). 